\title{
Design and Implementation of Digital Integral Interpolator Based on LabVIEW
}

\author{
Zeqing Yang ${ }^{1,}$, Zuojie Wang ${ }^{2, b}$, Kai Peng ${ }^{1, c}$, Weidong Yang ${ }^{1}$, Libing Liu ${ }^{1}$ \\ ${ }^{1}$ School of Mechanical Engineering, Hebei University of Technology, Tianjin 300130, China \\ ${ }^{2}$ Sinoma Technology \& Equipment Group CO.,LTD, Tianjin 300400, China \\ ayzq82@163.com, 'bangzuojie@tcdri.cn, ${ }^{\mathrm{c}}$ pengkai@hebut.edu.cn
}

Keywords: Numerical Control Interpolation; Digital Integral Method; Interpolator; LabVIEW

\begin{abstract}
In order to facilitate the students to visually understand digital integral interpolation principle and interpolation process, digital integral interpolator software system was developed using LabVIEW. The system is integrated with linear interpolation and arc interpolation modules, and every module mainly included the interpolation arithmetic initialization start function, interpolation speed control function and interpolation process real-time display function. The front and back panels of each module were designed based on LabVIEW platform, the programming flow charts were designed, and the system was tested comprehensively; the results showed that the interpolator can not only realize single quadrant linear and circular interpolation, but also can realize the span quadrant linear and circular interpolation; furthermore, it can simulate the specific interpolation process of the interpolation algorithm visually and intuitively, which can be used to assist teaching.
\end{abstract}

\section{Introduction}

The interpolation algorithm and interpolation principle of digital integral method is the most important part of the teaching contents in computer numerical control technology [1]. The existing interpolation simulation equipment is more expensive, and can not meet the experimental teaching. At present, the scholars research on the CNC interpolation simulation system based on FPGA [2,3] or VC [4], which realize the interpolation process, but the program code is not conducive to understand the interpolation process, and there is no interactivity. LabVIEW has the characteristics of visual programming environment and flowchart-style programming, high-efficient program compiler, flexible program debugging tools, powerful data processing and analysis library, so the digital integral interpolation software system was developed based on LabVIEW to assist teaching, not only vividly simulate single quadrant linear and circular interpolation, but also can achieve the function of across quadrants linear and circular interpolation, which can achieve good teaching effect and enhance the practice and interactivity in teaching contents.

\section{The interpolation principle of digital integral method}

Interpolation is an important function of the numerical control system, and interpolation is defined as the process of data intensive change, that is the numerical control system generates the basic straight line or arc and accomplishes the desired contour track fitting according to the input data of machining program and some methods [1].The Digital integral interpolation method is established based on digital integrator, the interpolation principle is that the displacements of tool along the coordinate axis are calculated using digital integral method, and the terminal point coordinate is accumulated respectively using accumulator when machining straight or arc, the corresponding coordinate feeds one step along the predetermined direction until it reaches the endpoint when the pulse is overflowed in the accumulator [1,4]. Digital integral method has high computing speed, pulse distribution uniform, easy to implement multi-axis linkage of all sorts of function curve in plane [4].

The linear interpolation principle of digital integral method 
Supposed there is a machining line OA in a plane $\mathrm{XOY}$ in the first quadrant, the starting point is the origin of the coordinates, and the coordinates of the end point is $\left(x_{e}, y_{e}\right)$,the synthetic feed speed of machining line is $v$, the speeds of moving point along $\mathrm{X}$ axis and $\mathrm{Y}$ axis are $v_{x}$ and $v_{y}$ respectively, the distance between starting point and ending point is $L$, the schematic diagram of digital integral method linear interpolation is shown in Fig.1, then $\frac{v_{x}}{x_{e}}=\frac{v_{y}}{y_{e}}=\frac{v}{L}=k$, where $\mathrm{k}$ is a constant. Then the micro-increments of the moving point along $\mathrm{X}$ axis and $\mathrm{Y}$ axis in $\Delta t$ are $\Delta x=v_{x} \Delta t=k x_{e} \Delta t$ and $\Delta y=v_{y} \Delta t=k y_{e} \Delta t$ respectively. Then the moving process of the moving point from original point to ending point can be regarded as an accumulative process of the coordinates of $\mathrm{X}$ and $\mathrm{Y}$ in the form of $k x_{e}$ and $k y_{e}$ in each unit time, and the moving point get the end point after accumulated for $m$ times, then it meets as followings: $x=\sum_{i=1}^{m} \Delta x=\sum_{i=1}^{m} k x_{e} \Delta t=m k x_{e}=x_{e}$ and $y=\sum_{i=1}^{m} \Delta y=\sum_{i=1}^{m} k y_{e} \Delta t=m k y_{e}=y_{e}$,it can be inferred that $m k=1$.Supposed there is $n$ bite register, the maximum allowed value of $x_{e}$ and $y_{e}$ is the maximum capacity value of register, that is $2^{n}-1$. In order to make sure that the number of pulse assigned is less than one, so it meets as followings: $\Delta x=k x_{e}=k\left(2^{n}-1\right)<1$ and $\Delta y=k y_{e}=k\left(2^{n}-1\right)<1$, it can be inferred that $k<\frac{1}{2^{n}-1}$, the accumulative times is $m=\frac{1}{k}=2^{n}$ for $k=\frac{1}{2^{n}}$ is required [1,6].

\section{The circular interpolation principle of digital integral method}

Supposed there is an inverse arc AB in a plane XOY in the first quadrant, the radius of arc is $\mathrm{R}$, the tangential velocity at the moving point is $v$, and the coordinates of the moving point $\mathrm{P}$ is $(x, y)$, the schematic diagram of digital integral method circular interpolation is shown in Fig.2.

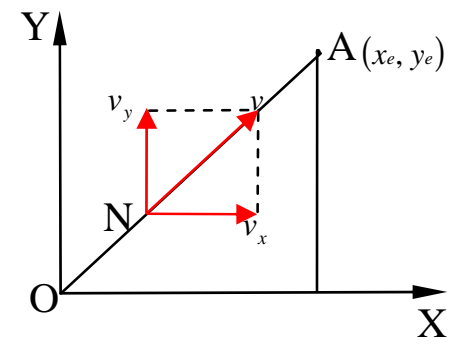

Fig.1 Schematic diagram of digital integral method linear interpolation

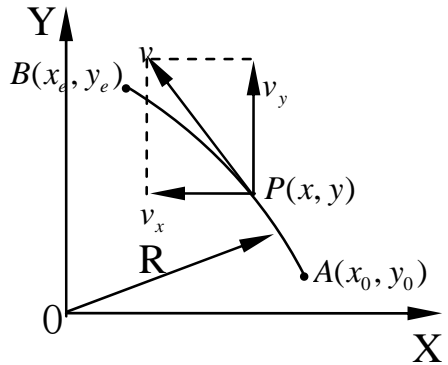

Fig.2 Schematic diagram of digital integral method inverse circular interpolation

Then $\frac{v}{R}=\frac{v_{x}}{y}=\frac{v_{y}}{x}=k$, where $v_{x}$ and $v_{y}$ are the speeds of moving point along $\mathrm{X}$ axis and $\mathrm{Y}$ axis respectively, and $\mathrm{k}$ is a constant. Then the micro-increments of the moving point along $\mathrm{X}$ axis and $\mathrm{Y}$ axis in $\Delta t$ are $\Delta x=-v_{x} \Delta t=-k y \Delta t$ and $\Delta y=v_{y} \Delta t=k x \Delta t$ respectively. The circular interpolation can be viewed as the accumulative process of the moving point coordinates in each unit time, if the integral accumulator overflows, the corresponding coordinate axis feeds one step in its direction, and then the moving point coordinates are modified and continue accumulating. The down-counters of coordinates $x$ and $y$ are expressed by $\left|x_{0}-x_{e}\right|$ and $\left|y_{e}-y_{0}\right|$, the corresponding down-counter minus one when the pulse is output from $x$ or $y$ integrator, and the coordinate get to the end and stop interpolation when the number of coordinate counter is zero in this direction, the circular interpolation is over when the number of all coordinate counters are zero.

\section{Design and implementation of digital integral interpolator}

\section{The composition of digital integral interpolator}

Modular design concept is adopted to modify and call the modular conveniently in designing digital integral interpolator. The function of linear and circular interpolation can be implemented separately in digital integral interpolator software, each function modular includes initialization modular (input of interpolation operation basic data, selection of clockwise arc or inverse arc, and 
starting the interpolation simulation process), interpolation speed control modular and interpolation process real-time display module.

\section{The front panel design of the digital integral interpolator}

The front panel of digital integral interpolator is designed according to the composition and function of CNC interpolator, including the initialization module, interpolation speed control module and interpolation process real-time display module. And the initialization module is used for inputting the interpolation operation basic data, including the start point coordinates and the end point coordinates, circle center, feed velocity, etc., and selecting clockwise arc or inverse arc, and starting the interpolation simulation process and displaying the system time. All of the functions are implemented by numerical input control and Boolean control. The interpolation speed control module is used for adjusting the interpolation speed $[7,8]$ and displaying the interpolation time. The interpolation process real-time display module is used for dynamic displaying the interpolation trajectory, changing in $\mathrm{X} / \mathrm{Y}$ feeding direction and displaying the interpolation process data, using XYGraph, waveform diagram, arrays, and numerical display control.

\section{Implementation of digital integral interpolator software}

The back panel block diagrams of digital integral linear interpolation (DILI) and circular interpolation (DICI) are shown in Fig.3 and Fig.4 respectively. The back panels programs were compiled according to the front panel design idea of digital integral interpolator and the interpolation flow diagrams. The start function of interpolator was achieved using while loop and Boolean switch, when the switch is true, the program jumps out of the while loop into the case loop and executes interpolation main program. The delay time function was adopted in while loop to reduce the CPU utilization and energy consumption, and the delay time is default set to $0.5 \mathrm{~s}$, which can also be modified according to needs. The basic data of the interpolation operation were input before the interpolator realizes operation; if you enter the wrong data or unreasonable data, the pop-up dialog box prompt the user to enter the correct data.

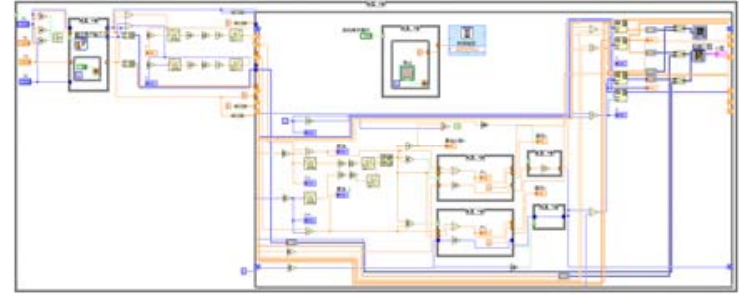

Fig.3 The back panel block diagram of DILI

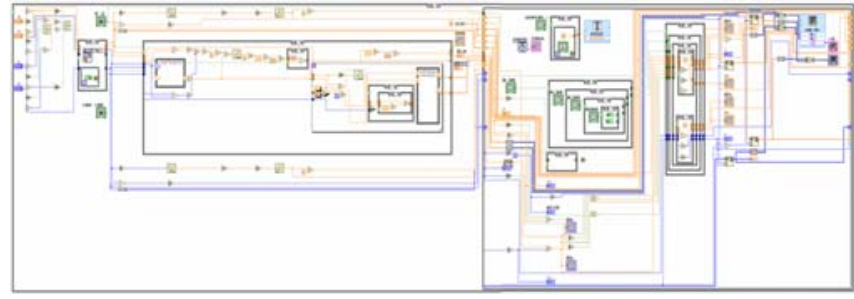

Fig.4 The back panel block diagram of DICI

The interpolation simulation process can be stimulated intuitively adopting time function to display interpolation time. The interpolation speed can be adjusted using time delay function to set the interval between two adjacent interpolation cycles. The single step interpolation control function is triggered to run an interpolation cycle, and the implementation of the interpolation process can be controlled by multiple triggering this button manually. The interpolation process real-time simulation display module was designed according to the flow diagram and interpolation step of different interpolation algorithm. The interpolation operation process was achieved by the data stream joined the ports, the nodes and block diagrams.

\section{Software testing of the digital integral interpolator}

The software testing is essential for digital integral interpolator software working, and it is also a necessary part of the normal working. First of all, every functional module is tested by inputting the start and end coordinates in different quadrant, and setting the interpolation feed speed, selecting the interpolation direction in circular interpolation. The tools of "show Error", "Step Into" and "Set Breakpoint" were used to debug the program and troubleshoot errors. The breakpoints were set to execute single-step in the program; the probe is set to observe the data changes in program running; or "Light up Operation" is used to observe the flow of the data stream. The system has good stability undergo a rigorous module testing and overall testing; the testing interfaces are shown in Fig.5. 

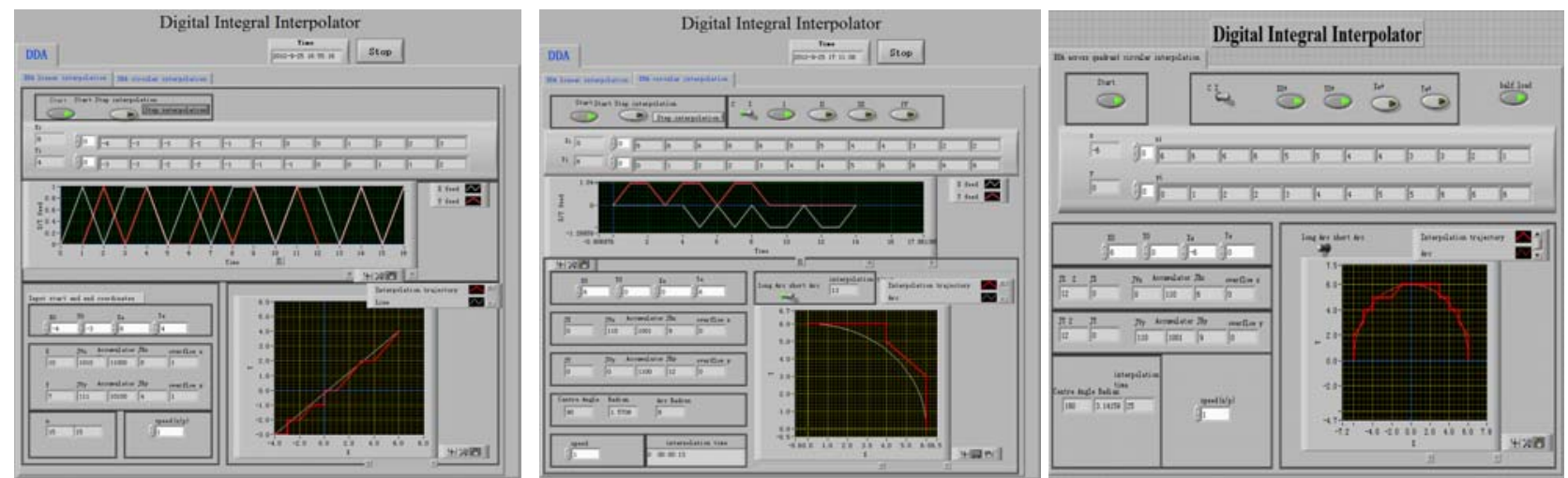

$\begin{array}{lll}\text { (a) Linear interpolation module } & \text { (b) Signal quadrant circular interpolation module } & \text { (c) across quadrant circular interpolation module }\end{array}$ Fig.5 Digital integration interpolation software testing interfaces

\section{Conclusion}

The overall framework and systematic process of the digital integral interpolator software system were designed based on the analysis of digital integral interpolation principle. Take linear interpolation and circular interpolation as examples, the interpolation function was realized in the LabVIEW, the interpolation process was executed through manually single-step, it also run automatically. Finally, the module test and overall test achieved satisfactory results. The system not only can be used for teaching and training students to understand the interpolation process and the implementation process of the classic interpolation algorithm intuitively, but also can be used for scientific research, which provides software platform for the improvements and applications of the elliptic interpolation and other complex curve interpolation algorithm. It has practical value and application prospect for in-depth research on digital integral interpolation algorithm.

\section{References}

[1] HUANG Jiashan: Computer Numerical Control Technology (Mechanical Industry Press, Beijing 2006).

[2] YANG Guang you, LI Liang, SU Xu wu, ZHOU Guozhu. Design and Implementation of DDA Interpolator Algorithm Based on FPGA [J].Journal of Hubei University of Technology, Vol.22, No.5, (2007), p.43- 46.

[3] ZHOU Bao ting,WANG Bai jun. A DDA arc interpolator for digital differential analyzer based on FPGA [J]. Electric Drive Automation, Vol.27, No.5, (2005), p.16-18.

[4] BAI Qinghua, JIANG Peigang, ZUO Yuhu. VC Implement of the DDA Circular Interpolation [J]. Machine Tool \& Hydraulics, No.7, (2006), p.219-220,254.

[5] ZHANGYongnian, ZHAO Dongbiao, LIU Kai, CHEN Sheng. A Uniformly Accelerated DDA (Digital Differential Analyzer) Interpolation Algorithm [J].Mechanical Science and Technology, Vol.29, No.12, (2010), p.1602-1605.

[6] WANG Zhongping, TIAN Zuohua. DDA 3D circular interpolation base on vector[J]. Machinery Design \& Manufacture,No.9, (2007), p.164- 165.

[7] R.V. Fleisig, A.D. Spence. A constant feed and reduced angular acceleration interpolation algorithm for multi-axis machining [J]. Computer-Aided Design, Vol.33, No.1, (2001), p.1-15.

[8] LIU Yuan, LI Hui, WANG Yongzhang. Realization of a 5-axis NURBS Interpolation with Controlled Angular Velocity [J]. Chinese Journal of Aeronautics, Vol.25, No.1, (2012), p. 124-130 